\title{
Different Occurrence Rates of Centrally Located Cytoplasmic Granulation in One Cohort Oocytes Show Distinctive Embryo Competence and Clinical Outcomes
}

\author{
Fangyuan Sun \\ Hangzhou Medical College \\ Jintao Cun \\ Kunming Women and Children Healthcare Hospital \\ Rongfang Huang \\ Kunming Women and Children Healthcare Hospital \\ Yunyan Chen \\ Kunming Women and Children Healthcare Hospital \\ Gideon Verwoerd \\ Hangzhou Medical College
}

Yuansong Yu ( $\nabla$ yuyuansong@eetop.com )

hang zhou yi xue yuan: Hangzhou Medical College https://orcid.org/0000-0002-0382-8298

\section{Research}

Keywords: Centrally located cytoplasmic granulation (CLCG), Oocytes, Intracytoplasmic sperm injection (ICSI), Frozen embryo transfer (FET), Clinical outcomes

Posted Date: September 21st, 2021

DOI: https://doi.org/10.21203/rs.3.rs-898506/v1

License: (c) (i) This work is licensed under a Creative Commons Attribution 4.0 International License. Read Full License 


\section{Abstract \\ Background}

Centrally located cytoplasmic granulation (CLCG) is a common cytoplasmic dysmorphism in human oocytes retrieved after controlled ovarian hyperstimulation $(\mathrm{COH})$. This study sought to achieve a better understanding of its formation and effects on clinical outcomes.

\section{Methods}

We retrospectively analyzed the data from 422 intracytoplasmic sperm injection (ICSI) treatment cycles. Three groups of patients were classified according to the rates of CLCG occurrence in one egg cohort, as partial (pCLCG) all (aCLCG) and no CLCG (control).

\section{Results}

The pCLCG group had a significantly lower Body Mass Index (BMI) and higher Anti-Mullerian hormone $(\mathrm{AMH})$ level compared to the control or aCLCG groups. Consistent with these distinctive features in the PCLCG group, fertilization and blastocyst formation rates were reduced significantly in the PCLCG group but not in the aCLCG group. Furthermore, the clinical outcomes in fresh embryo transfer cycles were dramatically reduced in the PCLCG group compared with the control group. However, in frozen/thawed cycles, all three clinical outcomes were significantly reduced in the aCLCG group but not in the pCLCG group.

\section{Conclusion}

We propose that PCLCG may reflect a specific population of patients, and that the CLCG structure is sensitive to cryopreservation.

\section{Background}

In the past forty years, assisted reproduction technology (ART) has been developed to a mature and major tool to solve infertility problems. However, it still puzzles embryologists that although cytoplasmic dysmorphisms frequently and repetitively occur in human oocytes collected after $\mathrm{COH}$, and oocyte morphology is commonly used as a predictor for embryo quality, the relationship between oocytes morphology and treatment outcomes is still unclear [1-5]. Centrally located cytoplasmic granulation (CLCG) is one of these abnormal phenotypes and was first described by Serhal et al. [6] as a larger, dark, spongy granular area in the cytoplasm. But until now it remains unclear which factors cause this morphological feature. It was proposed that CLCG might be a sign of oocyte cytoplasmic immaturity [79] and its formation could be related to patients' age or gonadotrophin (Gn) stimulation [10]. Moreover, it 
was reported that long term exposure to pesticide contaminated environment could cause the CLCG structure in human oocytes, and severely affect the fertility of local people $[11,12]$. But there are discrepancies among these studies and the evidence is not strong enough to be convincing $[5,7,10,13-$ 15]. Since there is a tendency for an abnormal phenotype to recur in the same patients $[1,15]$, it is likely that a correlation exists between the dysmorphism and patients' innate features.

There are also controversies in the literature regarding the effects of CLCG structure on fertilization, embryo development and clinical outcomes. Some studies showed that CLCG structure affected the fertilization and early embryo development $[12,16]$, the clinical pregnancy rate dramatically reduced and miscarriage rate increased when embryos derived from CLCG oocytes were transferred $[1,6,9,17]$ and these detrimental effects were related to chromosome aberrancies $[7,18]$, which indicated CLCG occurrence could be an indicator of genetic abnormalities $[7,18]$. However, more and more recent reports showed that CLCG structure did not affect fertilization and embryo development $[10,14,19]$ and also that clinical outcomes were not adversely affected $[14,20,21]$. Particularly in one recent study, CLCG oocytes were suggested to be treated as normal based on their precisely compared results [14]. However, few previous studies pay attention to the effects of CLCG structure on the pregnancy outcomes in frozen/thawed (FET) cycles, until a very recent study showed that freeze/thaw procedure could change the appearance of CLCG in some oocytes and clinical outcomes were disturbed with embryos derived from these oocytes [17]. Oocytes and embryo cryopreservation has been widely adopted in ART treatment, therefore a better understanding of the fate of embryos derived from CLCG oocytes would benefit the improvement of ART treatment.

We noticed that the different methodology of grouping patients might contribute to the obscure view of CLCG structures. In order to enhance understanding, we divided the patients into three groups according to the CLCG oocytes occurrence rate in a sibling oocytes cohort (control, no oocytes displayed CLCG; pCLCG, part of the oocytes cohort displayed CLCG; aCLCG, all oocytes in the cohort displayed CLCG), then analyzed the relevance of the women's clinical features to CLCG occurrence and compared the embryo competence and clinical outcomes between the different patient populations.

\section{Materials And Methods}

Statement: All experiments in this study were approved by the ethics committee of the Kunming Women and Children Healthcare Hospital and were performed in accordance with the relevant guidelines and regulations of the ethics committee of the Kunming Women and Children Hospital. All the patients involved in this study were fully informed and relevant consent forms were signed.

Patient selection and classification: All the data were collected from patients who accepted ICSI treatment in the Reproductive Medical Centre of the Kunming Women and Children Healthcare Hospital, during the period from June 2017 to November 2020. All patients involved in this study had definite indications for assisted reproductive treatment, including Fallopian tube problems, severe oligospermia or obstructive azoospermia. Only patients who had five or more oocytes in one retrieval cycle were included, 
which excluded low responder patients and avoided bias regarding embryo competence or clinical outcomes due to very limited numbers of oocytes.

The centrally located granulation was distinguished under the microscope during ICSI manipulation, healthy oocytes with clear and evenly distributed cytoplasm and CLCG oocytes with a centrally located, dark and spongy area were observed (Fig.1). We quantitatively defined the CLCG oocytes according to the method adopted by Yi et al. [14]. Briefly, both the whole oocytes area and central dark area at equator section were calculated with OCTAX (NaviLase, MTG) software as shown in Fig.1b, and oocytes with a central granulation area taking up to at least $25 \%$ of the whole oocytes were considered as CLCG oocytes. According to the occurrence pattern of CLCG, all the patients were divided into three groups i.e. control (no oocytes had a CLCG structure in the sibling cohort), pCLCG (a partial cohort of oocytes had a CLCG structure in the sibling cohort), aCLCG (all oocytes had a CLCG structure in the sibling cohort).

\section{Fertilization observation and embryo culture}

ICSI was performed normally on denuded oocytes, except for paying more attention to avoid interference with the granulation area during manipulation. Following the ICSI process, all oocytes were immediately moved into cleavage embryo culture medium (K-SICM, COOK), and cultured overnight. The next morning ( 17h after ICSI), fertilized oocytes with 2PN were distinguished under the microscope and moved into microdrops of cleavage medium individually. On Day 3, embryos that needed to continue culture to blastocysts, were moved into blastocyst culture medium (K-SIBM, COOK). Embryo morphology was observed daily and graded based on the criteria established by [23-25]. Embryos graded at or above 6C33 (Day 3 embryos) or 3BB (Day 5/6, blastocysts) were considered to be utilizable embryos and were transferred or frozen for later use.

\section{Embryo transferring and freezing}

For fresh embryo transfer (ET) cycles, one or two utilizable embryos scored 6C33 and above (Day 3) or 3BB and above (Day 5/6) were transferred into the uterine cavity under ultrasound guidance. Spare utilizable embryos were frozen using a vitrification kit (kitazato, Japan) according to the manufacturer's manual. For frozen/thawed embryo transfer (FET) cycles, the designated embryos were thawed using a thawing kit (kitazato, Japan) on the transfer day and the thawed embryos were kept in blastocyst culture medium in an incubator to recover for $\sim 2 \mathrm{~h}$ before they were transferred.

Endometrial preparation was performed according to clinical protocols in either artificial cycles or low dose gonadotropin ovulation induction cycles.

\section{Statistics}

All the data were analyzed with SPSS 20 software. The data for clinical outcomes and embryo degeneration rates were analyzed with the Chi-square test, and all the other analyses were performed using the student's t-test. A p-value of $<0.05$ was considered statistically significant. 


\section{Result}

\section{The distribution of patients groups and the correlations between group of patients and clinical features}

The data were collected and analyzed from a total of 422 cycles (402 patients), and the classified groups of control, pCLCG and aCLCG respectively included 253 cycles (247 patients), 84 cycles (81 patients) and 85 cycles (83 patients). The occurrence ratio of CLCG oocytes in the PCLCG group complied with a normal distribution and the median value of occurrence rate was $40 \%$ (Fig. 1c). For most of the analyzed factors, comparisons did not show significant differences among the three groups, i.e.control, pCLCG and aCLCG (Table 1). Surprisingly, pCLCG patients showed a lower BMI compared to the control $(p<0.05)$ and a higher AMH level compared to aCLCG group $(p<0.05)$.

Table 1

The correlation of CLCG occurrence with clinical features and a comparison between the three groups. * $\mathrm{p}<0.05$.

\begin{tabular}{|c|c|c|c|c|}
\hline & Control & PCLCG & aCLCG & $\begin{array}{l}\text { Multiple comparison } \\
\text { P value }\end{array}$ \\
\hline No. of cycles & 253 & 84 & 85 & - \\
\hline Age (year) & $32.55 \pm 0.33$ & $32.26 \pm 0.54$ & $33.68 \pm 0.52$ & NS \\
\hline BMI (kg/囚) & $22.80 \pm 0.20$ & $\begin{array}{l}21.92 \pm \\
0.30^{*}\end{array}$ & $22.71 \pm 0.39$ & $\begin{array}{l}\text { pCLCG versus control, } p= \\
0.03\end{array}$ \\
\hline $\mathrm{AMH}(\mathrm{ng} / \mathrm{mL})$ & $3.92 \pm 0.21$ & $4.16 \pm 0.41$ & $3.13 \pm 0.29$ & $\begin{array}{l}\text { pCLCG versus aCLCG, } p= \\
0.046\end{array}$ \\
\hline PCOS (case rate) & 0.13 & 0.17 & 0.12 & NS \\
\hline $\begin{array}{l}\text { Baseline day E2 } \\
(\mathrm{pg} / \mathrm{ml})\end{array}$ & $34.25 \pm 1.69$ & $37.53 \pm 3.11$ & $31.58 \pm 2.91$ & NS \\
\hline $\begin{array}{l}\text { Baseline day FSH } \\
(\mathrm{mlU} / \mathrm{ml})\end{array}$ & $5.25 \pm 0.14$ & $5.48 \pm 0.28$ & $5.21 \pm 0.28$ & NS \\
\hline Trigger day $E_{2}(p g / m l)$ & $\begin{array}{l}4833.0 \pm \\
192.9\end{array}$ & $\begin{array}{l}5463.8 \pm \\
369.1\end{array}$ & $\begin{array}{l}4778.3 \pm \\
333.5\end{array}$ & NS \\
\hline $\begin{array}{l}\text { Trigger day hCG } \\
(\mathrm{ng} / \mathrm{ml})\end{array}$ & $1.49 \pm 0.07$ & $1.36 \pm 0.08$ & $1.43 \pm 0.08$ & NS \\
\hline $\begin{array}{l}\text { Days of ovary } \\
\text { stimulation }\end{array}$ & $10.21 \pm 0.12$ & $10.65 \pm 0.22$ & $10.61 \pm 0.20$ & NS \\
\hline Total Gn doses (IU/L) & $\begin{array}{l}2018 \pm \\
59.83\end{array}$ & $\begin{array}{l}2039 \pm \\
85.35\end{array}$ & $\begin{array}{l}2058 \pm \\
73.42\end{array}$ & NS \\
\hline
\end{tabular}

The effect of CLCG structures on the oocytes maturation and embryo competence 
The oocytes maturation rates in the three groups i.e. control, pCLCG and aCLCG were $82.2 \%, 85.1 \%$ and $81.8 \%$ respectively, and no correlation was found between maturation rate and CLCG occurrence ( $p$ > 0.05 , Fig. 2). Fertilization and blastocyst formation rates were compromised significantly in the pCLCG group compared with the control group (Fig. 2, fertilization rate, $75.7 \%$ versus $81.7 \%, p<0.01$; and blastocyst formation rate, $62.9 \%$ versus $72.2 \%, p<0.05$ ), but the aCLCG group didn't show significant reduction in fertilization and blastocyst formation rates compared to the control group $(78.7 \%$ and $66.2 \%$ versus $81.7 \%$ and $72.2 \%, p>0.05$; Fig. 2). The utilizable embryo rate was comparable among all three groups $(p>0.05)$.

\section{The Clinical Outcomes In Et And Fet Cycles}

From a total of 138 fresh transfer cycles, the number of cycles in the control, pCLCG and aCLCG groups were 80, 31 and 27 respectively. As shown in Fig. 3a, the PCLCG group showed a dramatic reduction in the clinical outcomes of live birth plus ongoing pregnancy compared to the control group (29\% versus $55 \%, p=0.014)$. Reductions could also be seen in the clinical pregnancy and implantation rates, but without statistical significance. On the contrary, all three clinical outcomes in the aCLCG group were comparable to the control group.

From a total of 290 FET cycles, the number of cycles in the control, PCLCG and aCLCG groups were 157, 69 and 64 respectively. Contrary to the result in fresh transfer cycles, the aCLCG group showed a significant reduction in all three clinical outcomes i.e. clinical pregnancy rate, implantation rate and live birth plus ongoing rate compared to the control (Fig. 3 b, $42.2 \%, 27.3 \%$ and $34.4 \%$ versus $63.7 \%, 44.9 \%$ and $52.9 \%, p<0.01, p<0.01$ and $p<0.05$, respectively), but the clinical outcomes in the pCLCG group were comparable to the control group (Fig. 3b). We also compared the outcomes between fresh transfer cycles and FET cycles in the same group of patients (Fig. 3c-e), and noticed that in FET compared to fresh transfer cycles there was a tendency towards improved outcomes in the PCLCG group and worse outcomes in the aCLCG group. However these changes were not statistically significant.

\section{The heritage of CLCG structure in Cleavage embryo and its effects on the degradation of embryos subjected to freeze/thaw process}

In addition we monitored the heritage of the CLCG structure in fertilized oocytes and cleavage embryos derived from CLCG oocytes. The photos clearly showed that the central granulation area was distinctively retained after fertilization (Fig. 4a), and also can be distinguished in 4-cell stage (Fig. 4b) and 8-cell stage (Fig. 4c) embryos. However, due to the small size and compacted connection of the cells, the CLCG area could not be distinguished in blastocyst embryos (photo not shown). After we analyzed the degradation rates of all thawed embryos involved in FET cycles among the three groups of patients, the data clearly showed that embryos derived from CLCG oocytes are more sensitive to the freeze/thaw process as higher degradation rates were found in the PCLCG and aCLCG groups compared with controls ( $4.4 \%$ and $4.7 \%$ versus $0.6 \%, p<0.05$ ). 


\section{Discussion}

Oocytes morphology is commonly used to predict embryo competence and pregnancy outcomes in ART treatment, especially in ICSI cycles, as cytoplasmic structures are distinctively displayed in denuded oocytes. However, dysmorphisms always puzzle embryologists because of its repetitive occurrence $[1,15]$ and the controversies surrounding both the causes of formation and effects on clinical outcomes in previous studies $[3,7,10,13-15]$. CLCG is one of these abnormal phenotypes and previous studies proposed that CLCG might be a sign of oocytes cytoplasmic immaturity $[7,8,9]$ and its formation could be related to patients' age and Gn type [10]. A very recent case study showed that an antagonist protocol could improve the oocytes morphology with regards to CLCG, as well as the embryo quality, which indicates that the stimulation protocol is also related to the occurrence of CLCG for specific patients [15], Moreover, it was reported that long term exposure to a pesticide contaminated environment could cause CLCG in human oocytes, and severely affect the fertility of local people [11, 12]. Although there is no agreement regarding the causes of formation, we believe that exogenous stress and some patient innate features might be related to the manifestation of CLCG in human oocytes, because of repetitive occurrence in some patients and the effects of pesticide or Gn stimulation.

Most studies on the factors relevant to CLCG formation analyzed patients where CLCG was found in all oocytes in a sibling cohort $[7,14,18]$ or patients where at least one CLCG oocytes was found in one cohort [10]. In our preliminary observation, we found that there were always some patients repetitively showing either PCLCG or aCLCG. Whether these signatures of CLCG occurrence represent different populations of patients is unknown. Hence, in this study, we compared patients' features among three groups of patients (Control, pCLCG and aCLCG), and surprisingly, pCLCG patients had a significantly lower BMI and higher AMH level. Corresponding to this distinctive feature, the PCLCG group showed lower fertilization and blastocyst formation rates compared to the control group. In contrast, aCLCG patients did not show any difference from the control group in any of the analyzed clinical and embryo competence features, which is consistent to other studies $[10,13,14,16,19]$. Consistent with previous studies, aCLCG did not show any effects on all three clinical outcomes in fresh cycles $[14,20,21]$ but the clinical outcomes in PCLCG were dramatically worse. According to our results, we infer that the detrimental effects on the fertilization and blastocyst formation in the PCLCG group is probably not caused by the CLCG structure, and that the PCLCG group may represent a specific patient population distinctive from both control and aCLCG patients.

Organelles and cytoplasmic structures are sensitive to the freeze/thaw process [22, 26]. As a type of cytoplasmic structure and probably aggregations of organelles [1], CLCG structures could affect the clinical result after cryopreservation. A recent study showed different tolerance of vitrification and thawing among granulation patterns and thawed oocytes with appearance of smooth center and course edge of CLCG significantly affect the clinical outcomes [17]. Our tracing observation showed that the CLCG structure can be inherited by cleavage embryos and possibly blastocysts, which suggest that the embryos derived from CLCG oocytes may also have a higher sensitivity to the freeze/thaw process. An early study showed that freeze/thaw survival rate of embryos derived from CLCG oocytes was 
dramatically reduced [22]. Consistently, this study found a higher degradation of embryos after the freeze/thaw process in CLCG patients (Fig. 4d). However, much better survival rates compared to the early study [22] can be noticed, which reflects a general improvement of cryopreservation technique in current days.

Surprisingly, few studies pay attention to the effect of CLCG structure on the clinical outcomes in FET cycles, even though FET has been extensively adopted in ART treatment. For the first time, this study clearly showed a significant reduction of all three clinical outcomes in aCLCG patients in FET cycles compared to the control, and this compromising effect was not found in PCLCG patients. These results strongly suggest that CLCG structures may have long term detrimental effects on the embryos subjected to cryopreservation, even though the survival rate after freeze/thaw has been remarkably improved nowadays (Fig. 4d). The comparison of clinical outcomes between fresh transfer cycles and FET cycles among three groups of patients, revealed similar results in the control group, improved results in FET cycles in PCLCG patients, and compromised results in FET cycles in aCLCG patients. These distinctively different outcomes between the PCLCG and the aCLCG groups regarding fresh transfer and FET cycles further indicate that PCLCG patients probably represent an unique population with distinctive clinical features i.e. lower $\mathrm{BMI}$ and higher $\mathrm{AMH}$, as well as being more suitable for FET rather than fresh transfer. It has been proven that clinical outcomes can be improved by performing FET for polycystic ovary syndrome (PCOS) patients [27]. Although we didn't find a significant relevance between this group of patients with the diagnosis of PCOS (Table 1, p>0.05), a particular type of PCOS-lean PCOS with lower $\mathrm{BMI}$ has been proposed and well analyzed [28]. Hence, PCLCG patients with lower BMI and higher AMH might be related to the diagnosis of lean PCOS, which may explain the improvement of clinical outcomes in FET cycles for PCOS patients. However, further investigation need to be done to clarify the relationship between PCLCG and lean PCOS.

\section{Conclusion}

In conclusion, the presence of CLCG structures may have detrimental effects on embryos subjected to cryopreservation and adversely affect the clinical results in FET cycles, which suggests that an adjustment of the cyropreservation technique for embryos derived from CLCG oocytes is needed. Moreover, pCLCG patients may be a specific population with lower BMI and higher AMH, and an FAE (freeze all embryos) approach could be considered for this group of patients.

\section{Abbreviations}

\section{CLCG}

Centrally located cytoplasmic granulation; $\mathrm{COH}$ :Controlled ovarian hyperstimulation; ICSI Intracytoplasmic sperm injection; aCLCG:All oocytes with centrally located cytoplasmic granulation; pCLCG:Partial oocytes with centrally located cytoplasmic granulation; BMI:Body mass index; AMH:AntiMullerian hormone; FET:Frozen embryo transfer; ART:Assisted reproduction technology; Gn:Gonadotrophin; ET:Embryo transfer; PCOS:Polycystic ovary syndrome; FAE:Freeze all embryos. 


\section{Declarations}

\section{Ethics Approval}

This study was conducted by the approval of the Ethics Committee of Kunming City Maternal and Child Health Hospital (Approval number is (2021)002; Date 24/06/2021)

\section{Consent to Participate}

Not applicable

\section{Consent for Publication}

Not applicable

\section{Avaliability of data and materials}

The datasets generated and/or analyzed during the current study are not publicly available due to the ongoing further analysis and study based on the current study, but they are available from the corresponding author upon reasonable request

\section{Competing Interest}

The authors declare no competing interests

\section{Funding}

The author(s) received no public financial support for the research, authorship, and/or publication of this article

\section{Authors' Contributions}

YY and VG designed the study and wrote the manuscript, SF and CJ conducted the study and analyzed the data, $\mathrm{HR}$ and $\mathrm{CY}$ collected the data and involved some analyses. All the authors reviewed the manuscript

\section{Acknowledgements}

Not applicable

\section{References}

1. Meriano JS, Alexis J, Visram-Zaver S, Cruz M, Casper RF. Tracking of oocyte dysmorphisms for ICSI patients may prove relevant to the outcome in subsequent patient cycles. Hum Reprod.2001;16(10):2118-23. https://doi.org/10.1093/humrep/16.10.2118 
2. Otsuki J, Okada A, Morimoto K, Nagai Y, Kubo H. The relationship between pregnancy outcome and smooth endoplasmic reticulum clusters in MII human oocytes. Hum Reprod. 2004;19(7):15917. https://doi.org/10.1093/humrep/deh258

3. Balaban B, Urman B. Effect of oocyte morphology on embryo development and implantation. Reprod Biomed Online. 2006;12, 608-615. https://doi.org/10.1016/s1472-6483(10)61187-x

4. Montag M, Schimming T, Köster M, Zhou C, Dorn C, Rösing B, et al. Oocyte zona birefringence intensity is associated with embryonic implantation potential in ICSI cycles.Reprod Biomed Online. 2008;16: 239-244. https://doi.org/10.1016/s1472-6483(10)60580-9

5. Rienzi L, Vajta G, Ubaldi F. Predictive value of oocyte morphology in human IVF: a systematic review of the literature. Hum Reprod Update. 2010; 17:34-45. https://doi.org/10.1093/humupd/dmq029

6. Serhal PF, Ranieri DM, Kinis A, Marchant S, Davies M, Khadum IM.Oocyte morphology predicts outcome of intracytoplasmic sperm injection. Hum reprod. 1997;12: 1267-1270. https://doi.org/10.1093/humrep/12.6.1267

7. Kahraman S, Yakin K, Dönmez E, Samli H, Bahçe M, Cengiz G, et al. Relationship between granular cytoplasm of oocytes and pregnancy outcome following intracytoplasmic sperm injection. Hum reprod. 2000;15:2390-2393. https://doi.org/10.1093/humrep/15.11.2390

8. Ebner T, Moser M, Tews G. Is oocyte morphology prognostic of embryo developmental potential after ICSI ?. Reprod Biomed Online. 2006;12:507-512. https://doi.org/10.1016/s1472-6483(10)62006-8

9. Hu J, Molinari E, Darmon S, Zhang L, Patrizio P, Barad DH, et al. Predictive value of cytoplasmic granulation patterns during in vitro fertilization in metaphase II oocytes: Part I, poor-prognosis patients. Fertil Steril. 2021; Aug;116(2):431-443. https://doi.org/10.1016/j.fertnstert.2021.02.022

10. Fancsovits P, Tothne ZG, Murber A, Rigo JJr, Urbancsek J. Importance of cytoplasmic granularity of human oocytes in in vitro fertilization treatments.Acta Biol Hung. 2012;63:189-201. https://doi.org/10.1556/ABiol.63.2012.2.3

11. Merviel P, Cabry R, Chardon K, Haraux E, Scheffler F, Mansouri NB, et al. Impact of oocytes with CLCG on ICSI outcomes and their potential relation to pesticide exposure. J Ovarian Res. 2017; 10:42. https://doi.org/10.1186/s13048-017-0335-2

12. Cabry R, Merviel P, Madkour A, Lefranc E, Scheffler F, Desailloud R, et al. The impact of endocrine disruptor chemicals on oocyte/ embryo and clinical outcomes in IVF. Endocr Connect. 2020;9:R134R142.https://doi.org/10.1530/EC-20-0135

13. Ebner T, Moser M, Shebl O, Sommergruber M, Yaman C, Tews G. Blood clots in the cumulus-oocyte complex predict poor oocyte quality and post-fertilization development. Reprod Biomed Online. 2008: 16: 801-807. https://doi.org/10.1016/s1472-6483(10)60145-9

14. Yi XF, Xi HL, Zhang SL, Yang J. Relationship between the positions of cytoplasmic granulation and the oocytes developmental potential in human. Sci Rep. 2019;9:7215. https://doi.org/10.1038/s41598-019-43757-8

15. Lan KC, Chen YC, Lin YC, Tsai YR. Gonadotrophin-releasing hormone agonist triggering may improve central oocyte granularity and embryo quality.Zygote. 2020; 28: 337-343. 
https://doi.org/10.1017/S0967199420000106

16. Rienzi L, Ubaldi FM, lacobelli M, Minasi MG, Romano S, Ferrero S, et al. Significance of metaphase II human oocyte morphology on ICSI outcome. Fertil Steril. 2008;90:1692-1700. https://doi.org/10.1016/j.fertnstert.2007.09.024

17. Hu J, Molinari E, Darmon SK, Zhang L, Patrizio P, Barad DH, et al. Predictive value of cytoplasmic granulation patterns during in vitro fertilization in metaphase II oocytes: part II, donor oocyte cycles. Fertil Steril. 2021; Jul 19:S0015-0282(21)00526-4. https://doi.org/10.1016/j.fertnstert.2021.06.026

18. Yakin K, Balaban B, Isiklar A. Urman B. Oocyte dysmorphism is not associated with aneuploidy in the developing embryo. Fertil Steril. 2007;88: 811-816. https://doi.org/10.1016/j.fertnstert.2006.12.031

19. Wilding M, Di Matteo L, D'Andretti S, Montanaro N, Capobianco C, Dale B. An oocyte score for use in assisted reproduction. J Assist Reprod Genet. 2007;24:350-358. https://doi.org/10.1007/s10815007-9143-8

20. Balaban B, Urman B, Sertac A, Alatas C, Aksoy S, Mercan R. Oocyte morphology does not affect fertilization rate, embryo quality and implantation rate after intracytoplasmic sperm injection. Hum Reprod. 1998;13: 3431-3443. https://doi.org/10.1093/humrep/13.12.3431

21. Esfandiari N, Burjaq H, Gotlieb L,Casper RF. Brown oocytes: implications for assisted reproductive technology. Fertil Steril. 2006;86:1522-1525. https://doi.org/10.1016/j.fertnstert.2006.03.056

22. Balaban B, Ata B, Isiklar A, Yakin K, Urman B. Severe cytoplasmic abnormalities of the oocyte decrease cryosurvival and subsequent embryonic development of cryopreserved embryos. Hum Reprod. 2008;23:1778-1785. https://doi.org/10.1093/humrep/den127

23. Cutting R, Morroll D, Roberts SA, Pickering S, Rutherford A. Elective single embryo transfer: guidelines for practice British Fertility Society and Association of Clinical Embryologists. Hum Fertil. 2008; 11: 131-146. https://doi.org/10.1080/14647270802302629

24. Gardner DK, Schoolcraf WB. Culture and transfer of human blastocysts. Curr Opin Obstet Gynecol. 1999b;11:307-311.https://doi.org/10.1097/00001703-199906000-00013

25. Gardner DK, Lane M, Stevens J, Schlenker T, Schoolcraf WB. Blastocyst score affects implantation and pregnancy outcome: towards a single blastocyst transfer. Fertil Steril. 2000 Jun;73(6):11558. https://doi.org/10.1016/S0015-0282(00)00518-5

26. Khalili MA, Shahedi A, Ashourzadeh S, Nottola SA, Macchiarelli G, Palmerini MG.Vitrification of human immature oocytes before and after in vitro maturation: a review. J Assist Reprod Genet. 2017;34: 1413-1426. https://doi.org/10.1007/s10815-017-1005-4

27. Chen ZJ, Shi $Y$, Sun $Y$, Zhang B, Liang $X$, Cao $Y$, et al. Fresh versus Frozen Embryos for Infertility in the Polycystic Ovary Syndrome. N Engl J Med. 2016; 375:523-533. https://doi.org/10.1056/NEJMoa1513873

28. Toosy S, Sodi R, Pappachan JM. Lean polycystic ovary syndrome (PCOS): an evidence-based practical approach. J Diabetes Metab Disord. 2018; 17:277-285. https://doi.org/10.1007/s40200018-0371-5 
Figures
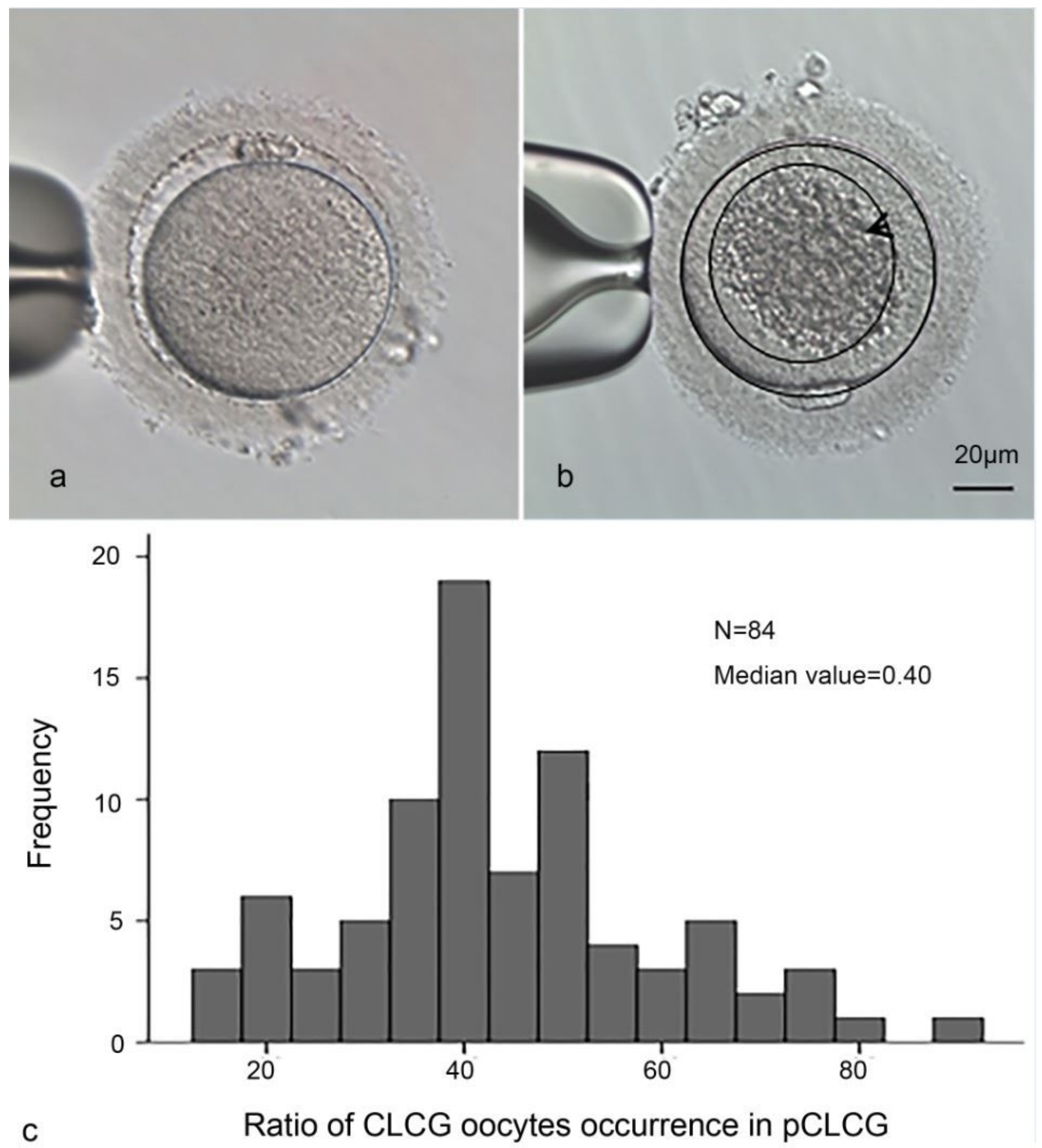

Figure 1

Description of CLCG oocyte and the distribution of its' occurrence ratio in the PCLCG group. a, Healthy oocytes with evenly distributed and clear cytoplasmic appearance; b, CLCG oocytes with dark, spongy, granulated area displayed in the center (as indicated with an arrow). The entire oocytes area and the 
central granulated area is calculated as circled. c, the normal distribution curve of CLCG oocytes occurrence ratios in the pCLCG group ( $n=84$, median ratio is $40 \%$ )

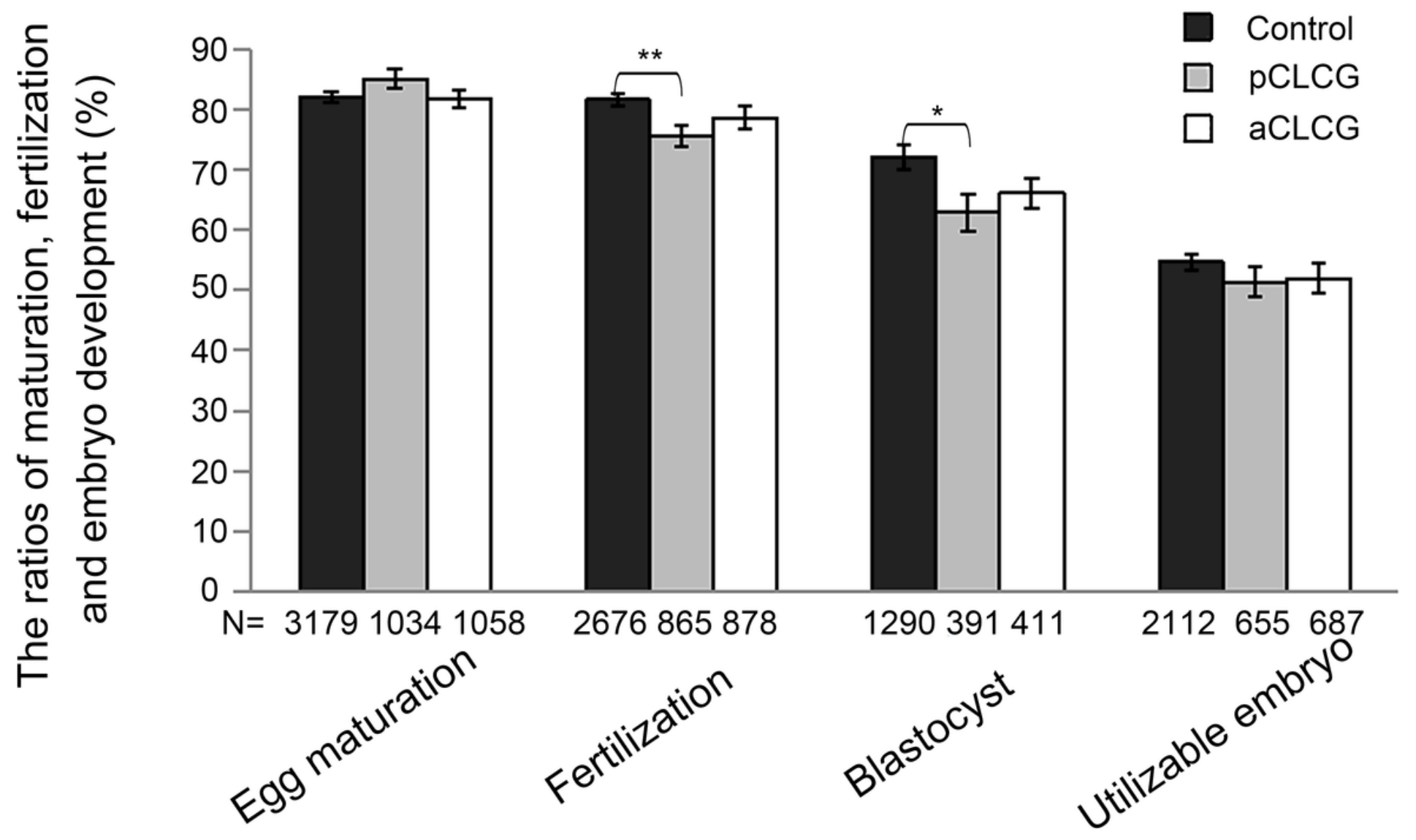

Figure 2

The correlation of CLCG occurrence with oocytes maturation, fertilization rate and embryo competence. * $p<0.05 ; * \star p<0.01$ 

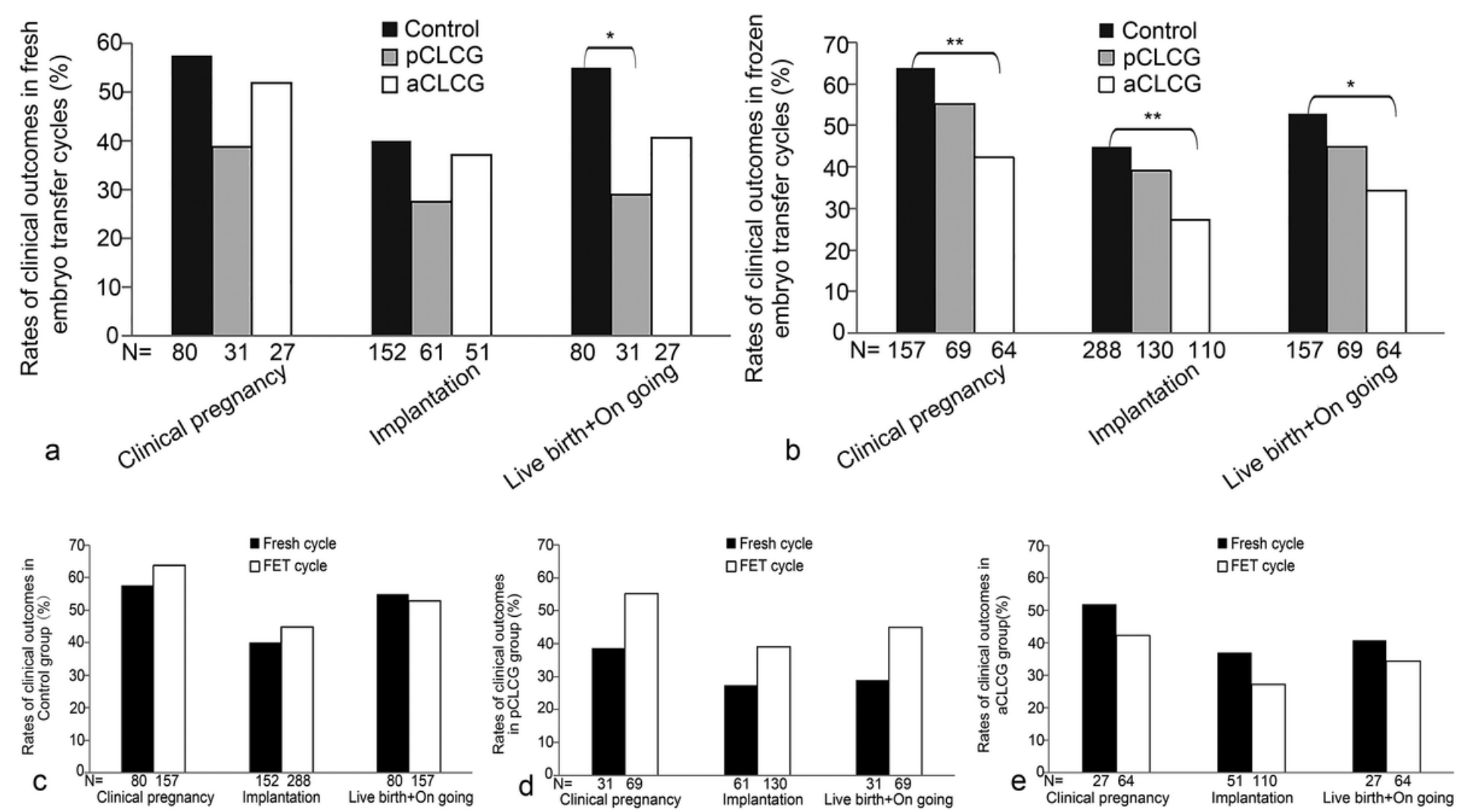

Figure 3

The clinical outcomes in fresh embryo transfer cycles (a) and frozen embryo transfer cycles (b) in the control, $p C L C G$ and aCLCG groups ( ${ }^{*} p<0.05 ; * \star p<0.01$ ). Although, the clinical outcomes in control groups(c) between ET and FET are quite comparable, a tendency towards improved clinical outcomes were found in PCLCG patients (d), and worse clinical outcomes in aCLCG patients(e) in FET cycles compared to ET cycles $(p>0.05)$ 


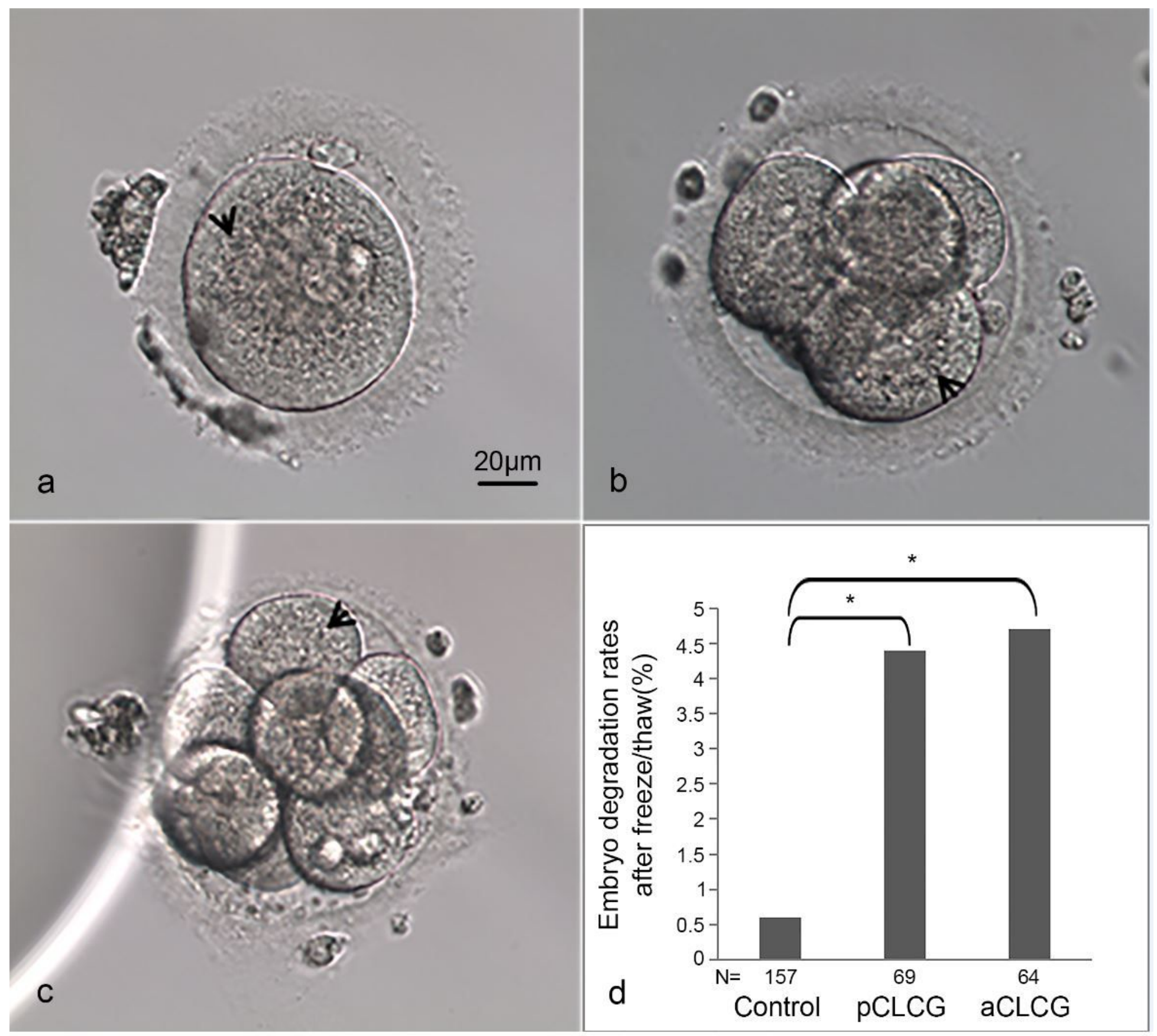

Figure 4

The heritage of CLCG structure in cleavage embryos and the degradation of embryos after the freeze/thaw process. The CLCG structure can still be distinguished after fertilization (PN embryo, a), at the D2 stage (4-cell embryo, b) and at the D3 stage (8-cell embryo, c). Embryos derived from CLCG oocyte showed a higher degradation rate after the freeze/thaw process in both the PCLCG and aCLCG groups (d, $\left.{ }^{*} \mathrm{p}<0.05\right)$ 